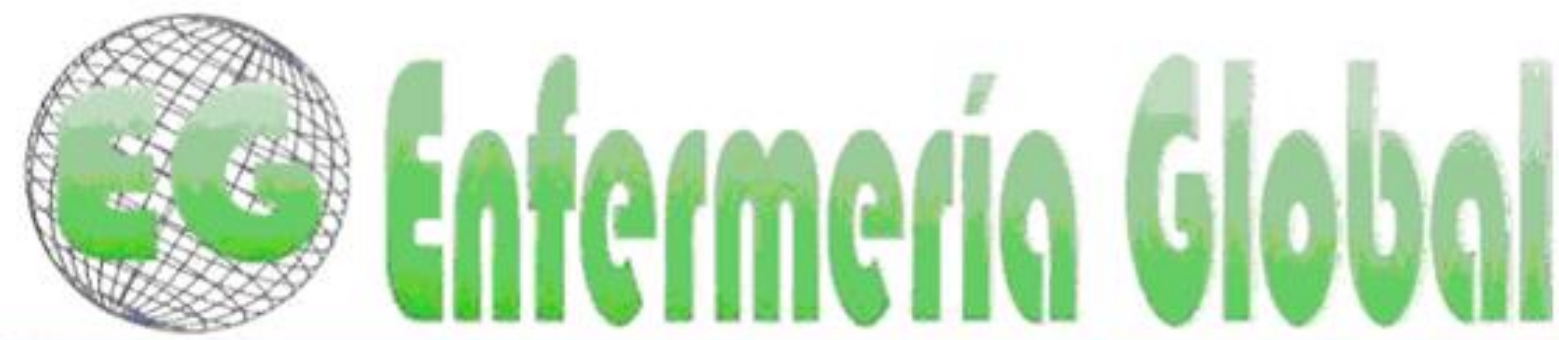

ISSN 1695-6141

$N^{\circ} 43$

Revista electrónica trimestral de Enfermería

Julio 2016

www.um.es/egloball

\title{
CLÍNICA
}

\section{Calidad de vida en pacientes con insuficiencia renal crónica en hemodiálisis}

Qualidade de vida de pacientes com insuficiência renal crônica em tratamento hemodialítico Quality of life of patients with chronic kidneydisease undergoing hemodialysis

\section{*Costa, Gabrielle Morais Arruda **Pinheiro, Maria Berenice Gomes}

\section{Nascimento ${ }^{* * *}$ Medeiros, Soraya Maria de ${ }^{* * *}$ Costa, Raphael Raniere de Oliveira ${ }^{* * * * *}$ Cossi, Marcelly Santos}

*Enfermera. Miembro del Grupo de Investigación Caleidoscopio de la Educación en Enfermería de la Universidad Federal de Rio Grande do Norte - UFRN. Natal, Rio Grande do Norte. **Enfermera. Máster en Salud Comunitaria. Docente de la Universidad Federal de Campina Grande - UFCG. Campina Grande, Paraíba ${ }^{* * * D}$ Doctora en Enfermería. Docente de UFRN, líder del Grupo de Investigación Caleidoscopio de la Educación en Enfermería de la UFRN. ${ }^{* * * *}$ Máster en Enfermería. Doctorando del Programa de PosGraduación en Enfermería de la UFRN. Miembro del Grupo de Investigación Caleidoscopio de la Educación en Enfermería - UFRN. E-mail: raphaelraniere@hotmail.com ******Enfermera. Alumna de Máster del Programa de PosGraduación en Enfermería de la UFRN. Miembro del Grupo de Investigación Caleidoscopio de la Educación en Enfermería - UFRN. Brasil.

Palabras clave: Calidad de Vida; Hemodiálisis; Insuficiencia renal

Palavras chave: Qualidade de Vida; Hemodiálise; Insuficiência renal.

Keywords: Quality of life; Hemodialysis; kidney failure..

\section{RESUMEN}

Esta investigación tuvo como objetivo evaluar la calidad de vida de los pacientes con enfermedad renal crónica en hemodiálisis, así como caracterizarlos, identificar los factores que afectan e influyen en la misma. Se trata de un estudio de campo, descriptivo, transversal y cuantitativo realizado en un centro de hemodiálisis en el Alto Sertão da Paraíba, municipio Cajazeiras. Se utilizó un cuestionario sociodemográfico y el WHOQOL-BREF para evaluar los puntajes promedio y la calidad de vida de los participantes, así como la prueba de correlación de Pearson entre las variables obtenidas. La muestra estuvo conformada por 39 sujetos. La investigación incluyó a pacientes de ambos sexos: $54 \%$ hombres y $46 \%$ mujeres y tiene, con respecto a la edad, alta prevalencia de más de 51 años. En general, el 
dominio más afectado entre los participantes fue el dominio físico (DF), con un promedio de 59.44, y el mejor conservado fue el dominio social (DS), con un promedio de 72.87 , reflejado en la Calidad de Vida (QOL) de los pacientes con IR, que mostró un promedio de 64.96. A través del test de correlación de Pearson, hubo una relación significativa entre los diversos ámbitos en los que todos los dominios mostraron una alta correlación con la calidad de vida. Llegamos a la conclusión de que los pacientes con enfermedad renal crónica en hemodiálisis tienen una calidad de vida regular, y el conocimiento de los profesionales sobre este tema es de suma importancia para alcanzar una optimización en el cuidado de los pacientes con IRC sometidos a hemodiálisis.

\section{RESUMO}

Esta pesquisa objetivou avaliar a qualidade de vida dos pacientes portadores de doença renal crônica em tratamento hemodialítico, bem como caracterizá-los, identificar os fatores que interferem e influenciam na mesma. Trata-se de um estudo de campo, descritivo, transversal, com abordagem quantitativa realizada em um centro de hemodiálise do Alto Sertão da Paraíba, município de Cajazeiras. Foi utilizado um questionário sócio-demográfico e o Whoqol-bref para avaliar a média dos domínios e da qualidade de vida dos participantes, bem como o teste de Correlação de Pearson entre as variáveis obtidas. A amostra foi composta por 39 sujeitos. A pesquisa contou com pacientes de ambos os sexos: $54 \%$ do sexo masculino e $46 \%$ do sexo feminino e possui, com relação a faixa etária, alta prevalência para maiores de 51 anos. De modo geral, o domínio mais prejudicado entre os participantes foi o físico (DF), com média de 59,44, e o mais preservado foi o domínio social (DS), com média de 72,87, refletindo na Qualidade de Vida (QV) dos portadores de IR, que apresentaram uma média de 64,96. Através do teste de Correlação Pearson observou-se relação de significância entre os diversos domínios, onde todos os domínios apresentaram uma correlação alta com a QV. Conclui-se que os pacientes com IRC em tratamento hemodialítico possuem uma qualidade de vida regular, e que o conhecimento dos profissionais acerca deste assunto é primordial para que alcance uma otimização na assistência aos portadores de IRC em tratamento hemodialítico.

\section{ABSTRACT}

This research aimed to evaluate the quality of life of patients with chronic kidney disease undergoing hemodialysis treatment, as well as to characterize them, identify the factors that affect and influence them. This is a field study, descriptive, cross-sectional and quantitative approach performed in a hemodialysis center in Alto Sertão da Paraíba, Cajazeiras municipality. A socio-demographic questionnaire and the WHOQOL-BREF were used to evaluate the average domains and quality of life of participants, as well as the Pearson correlation test between variables obtained. The sample consisted of 39 subjects. The research included patients of both genders: $54 \%$ male and $46 \%$ female and has, concerning age group, high prevalence for older than 51 years old. In general, the most affected domain among the participants was the physical domain (FD), averaging 59.44, and the best preserved was the social domain (SD), averaging 72.87, reflecting on Quality of Life (QOL) of patients with RI, which showed an average of 64.96. Through the Pearson correlation test, there was a significant relationship between the various domains, where all domains showed a high correlation with QOL. It was concluded that patients with CKDundergoing hemodialysis treatment have a regular quality of life, and the knowledge of the professionals about this issue is paramount to reach an optimization in the care of patients with CKD undergoing hemodialysis treatment.

\section{INTRODUCCIÓN}

Las enfermedades crónicas han recibido mayor atención de los profesionales de salud por presentar índice de altas tasas de morbimortalidad, convirtiéndose así en una grane preocupación para el campo de la salud pública. Entre las varias enfermedades crónicas que afectan a la población, la Insuficiencia Renal Crónica (IRC) es considerada una patología sin expectativa de cura, de evolución rápida y progresiva, desencadenando diversas reacciones para los pacientes, además de comprometer la calidad de vida (QV) ${ }^{(1-2)}$. 
Esta enfermedad está presente en un gran número de personas y presenta una evolución gradual e irreversible, generando complicaciones y perjuicios para los pacientes portadores. Se trata de una enfermedad cuyo principal tratamiento es la diálisis.

La IRC y el tratamiento dialítico desencadenan diversas situaciones para el paciente, comprometiendo varios aspectos relacionados con la salud. Las condiciones de tratamiento y la evolución crónica de la enfermedad limitan a los portadores de IRC y son, por tanto, factores agresores que desencadenan estrés, aislamiento social, así como limitaciones a la posibilidad de locomoción y paseos, disminución de las actividades físicas, dependencia y sentimiento de miedo e inseguridad con relación a la salud y bienestar. Por tanto, la CV es un factor de extrema importancia porque interfiere directamente en la efectividad de tratamientos e intervenciones en el área de salud $^{(1-3)}$.

Incluso con los avances tecnológicos y terapéuticos alcanzados hasta hoy con el objetivo de mejorar la condición clínica y aumentar la supervivencia del portador de IRC, su nivel de la calidad de vida continúa en declive ${ }^{(4)}$.

Sabiendo que la asistencia de enfermería necesita constantemente de modificaciones para atender a las necesidades individuales de la población, es necesario, y de extrema importancia, avanzar en el campo de la investigación para mejorar la calidad de la asistencia de enfermería y, consecuentemente, la CV del individuo ${ }^{(5)}$.

Por ese motivo se optó por realizar este estudio, con el objetivo de evaluar la CV de los pacientes portadores de IRC en tratamiento hemodialítico del Alto Sertão da Paraíba, Brasil. Así como, caracterizarlos en cuanto a las variables sociodemográficas, averiguar los factores que interfieren en las actividades diarias de los pacientes e identificar la correlación entre la calidad de vida global y los diversos dominios del WHOQOL-Bref.

\section{MATERIALES Y MÉTODOS}

Se trata de un estudio descriptivo, transversal, con un abordaje cuantitativo. Este estudio se realizó en el Centro de Hemodiálisis de Cajazeiras ( $\mathrm{CHC}$ ), estado de Paraíba, Brasil. El Centro de Hemodiálisis de Cajazeiras está agregado a la estructura física del Hospital Regional de Cajazeiras (HRC) y es de pequeño tamaño. El CHC atiende15 municipios circundantes, y cuenta con un equipo compuesto por médicos, enfermeros y técnicos de enfermería que son responsables del proceso de diálisis y del mantenimiento del estado de salud del paciente durante la sesión.

La muestra inicial estuvo compuesta por 50 pacientes con IRC haciendo tratamiento hemodialítico en el CHC. Fueron incluidos en la investigación los portadores de IRC mayores de 18 años, de ambos sexos y que estaban en tratamiento hemodialítico más de 6 meses. Fueron excluidos los pacientes que no aceptaron, de forma voluntaria, firmar el Término de Consentimiento Libre Esclarecido (TCLE), así como los portadores de trastornos mentales, o aquellos que, en el acto de la colecta de datos, presentaron un descenso del nivel de consciencia. Fueron excluidos también los pacientes que no comparecieron en la unidad de hemodiálisis durante el periodo estipulado para la colecta. Aplicados los criterios de inclusión, la muestra final estuvo compuesta por 39 sujetos. 
La investigación fue aprobada por el Comitê de Ética en Investigación de la Universidad Federal de Campina Grande, Paraíba, Brasil, bajo protocolo n 338.082, y desarrollada obedeciendo y respetando todos los presupuestos exigidos por la Resolución 196/96 del Consejo Nacional de Salud (CNS).

Como instrumentos de colecta de datos, se utilizaron dos instrumentos: un cuestionario dirigido a la caracterización sociodemográfica de los participantes y otro referente a análisis de calidad de vida, Whoqol-bref $f^{(7)}$.

Los datos obtenidos fueron digitados en planilla electrónica del programa Excel for Windows, versión 2010, conferidos, corregidos e importados para el programa Statistical Package for the Social Sciences (Programa SPSS), versión 17.0, para análisis estadístico.

\section{RESULTADOS}

La tabla 1 representa los datos sociodemográficos de los pacientes con IRC en Tratamiento hemodialítico en el municipio de Cajazeiras.

Tabla 1: Distribución de los participantes de la investigación en cuanto al género, grupo de edad, estado civil, escolaridad, ocupación, total de personas con quien vive y tiempo de tratamiento hemodialítico.

\begin{tabular}{lll}
\hline VARIABLES & $\boldsymbol{F}$ & $\boldsymbol{\%}$ \\
\hline Género & & \\
& & 46 \\
Femenino & 18 & 54 \\
Masculino & 21 & \\
& & \\
Edad (años) & & 10,25 \\
& 04 & 33,34 \\
18 a 30 años & 13 & 56,41 \\
31 a 50 años & 22 & \\
51 o más años & & \\
& & 23,08 \\
Estado civil & 09 & 66,67 \\
& 26 & 10,25 \\
Soltero & 04 & \\
Casado & & \\
Viudo & & \\
& & \\
Escolaridad & 06 & 17 \\
No sabe leer ni escribir & 02 & 53 \\
Alfabetizado & 19 & 53 \\
Enseñanza Básica Incompleta & 02 & 37 \\
Enseñanza Básica Completa & 01 & \\
Enseñanza Media Incompleta & 06 & \\
Enseñanza Media Completa & 01 & \\
Enseñanza Técnica & 02 & \\
Enseñanza Superior & & \\
\hline
\end{tabular}




\begin{tabular}{|c|c|c|}
\hline Ocupación & & \\
\hline Beneficiario & 17 & 44 \\
\hline Jubilado & 20 & 51 \\
\hline Pensionista & 02 & 5 \\
\hline $\begin{array}{l}\text { ¿Cuántas person } \\
\text { usted? }\end{array}$ & & \\
\hline Solo & 01 & 2,56 \\
\hline 1 persona & 07 & 17,95 \\
\hline 2 personas & 07 & 17,95 \\
\hline 3 personas & 13 & 33,34 \\
\hline 4 personas & 05 & 12,82 \\
\hline 5 o más personas & 06 & 15,38 \\
\hline
\end{tabular}

$\begin{array}{lll}\begin{array}{l}\text { Tiempo } \\ \text { Hemodiálisis }\end{array} & \text { realiza } & \\ \\ \text { Hasta 4 años } & 23 & 58,97 \\ \text { Más de 4 años } & 16 & 41,03\end{array}$

$\begin{array}{lll}\text { TOTAL } & 39 & 100\end{array}$

Fuente: Investigación directa, 2013

Se observa que, en cuanto al género y grupo de edad, es posible analizar que el índice de hombres que realizan el tratamiento alcanza un porcentual de $54 \%$; superior al de las mujeres, que representan $46 \%$ de los participantes. Se observa también que, con el aumento del grupo de edad, hay una elevación en el índice de prevalencia de la IRC en los pacientes, llegando a los siguientes resultados: entre 18 y 30 años, se obtiene un porcentual de $10,25 \%$; de 31 a 50 años $33,34 \%$ y de 51 años en adelante hay una prevalencia de $56,41 \%$.

Es posible observar que tales datos no presentan contradicción cuando comparados para detectar hemodiálisis en el estudio realizado por la SBN, en el cual se constató que el número de hombres que realizan el tratamiento es superior al de las mujeres; así como el grupo de edad más prevalente en el tratamiento va de 19 a 64 años, aumentando la incidencia con el avance de la edad ${ }^{(8)}$.

Se observó que, en relación al estado civil, la mayoría de la población entrevistada está oficialmente casada $(66,67 \%)$ y vive con sus compañeros.

De esa forma, fue posible concluir que los participantes de la investigación que estaban casados no se sentían solos en el enfrentamiento de la enfermedad, superando los obstáculos y desafíos impuestos por las limitaciones físicas derivadas de esta.

De acuerdo con los datos de la investigación, es posible evaluar que la mayoría de la población posee algún grado de instrucción, contando solo con $17 \%$ que no saben leer ni escribir y $5 \%$ tienen solo la alfabetización. 
Cabe observar que, en el ámbito ocupacional/profesional de cada participante, ninguno se encuentra en actividad laboral. El estudio indica que $44 \%$ reciben ayuda enfermedad, o sea, reciben el beneficio específico para los portadores de IRC dependientes del tratamiento de hemodiálisis; $51 \%$ son jubilados y $5 \%$ son pensionistas.

La IRC es una enfermedaad incapacitante en la dimensión profesional, o sea, impone dificultades y limitaciones físicas que anulan, la mayoría de las veces, la aptitud para el trabajo. En este estudio, específicamente, $100 \%$ de los participantes están desempleados.

En cuanto a la cuestión salarial, la falta de empleo en este grupo genera conflictos. En los relatos se identificó una reducción en el nivel de vida por el hecho de gozar solamente de beneficios/jubilación/pensión, siendo tal situación un factor importante en la alteración de la CV.

Otro aspecto social analizado en esta investigación fue la observación de la cantidad de personas que vivían solas o con otras personas, formando parte de la convivencia social familiar. Se observó que solo $2,56 \%$ de ellas vivían solas, estadística que corresponde a 01 persona; en cuanto a los demás, 17,95\% viven con 01002 personas; 33,34\% viven co 03 personas, constituyendo la mayor parte de la población; 12,82\% viven con 04 personas y 15,38\% viven con 05 o más personas.

Para analizar el tiempo de tratamiento en hemodiálisis, fueron incluidos aquellos que se tratan desde hace más de seis meses, considerando que dentro de este periodo de tiempo cambios y variaciones en la calidad de vida serían más evidentes. Se dividió, entonces, a los pacientes que realizan tratamiento de seis meses a 04 (cuatro) años, comprendiendo un valor estadístico de 58,97\%; y los que realizan hemodiálisis hace más de 04 (cuatro) años, 41,03\%.

En esta etapa, los datos fueron analizados de acuerdo con el instrumento de colecta Whoqol-Bref, específicos para calidad de vida, aplicado a los portadores de IRC en tratamiento de hemodiálisis del municipio de Cajazeiras, Estado de Paraíba.

El instrumento utilizado tiene cuatro dominios: físico, psicológico, social y ambiental; presentados en la tabla abajo:

Tabla 2. Puntuaciones obtenidas en el WHOQOL-bref de pacientes en hemodiálisis (N=39). Cajazeiras-PB, 2013.

\begin{tabular}{|c|c|c|c|c|c|c|}
\hline \multicolumn{7}{|c|}{ Estadística } \\
\hline & & $\mathrm{DF}$ & $\mathrm{DP}$ & DS & DA & QV \\
\hline \multirow[t]{2}{*}{$\mathrm{N}^{\mathrm{o}}$} & Válidos & 39 & 39 & 39 & 39 & 39 \\
\hline & Perdidos & 0 & 0 & 0 & 0 & 0 \\
\hline
\end{tabular}




\begin{tabular}{l|r|r|r|r|r}
\hline Media & 59,44 & 65,39 & 72,87 & 62,19 & 64,96 \\
& & & & & \\
\hline Desvío Estandar & 12,913 & 9,900 & 14,394 & 9,398 & 8,425 \\
& & & & & \\
\hline Mínimo & 32 & 38 & 25 & 41 & 44 \\
\hline Máximo & 89 & 92 & 100 & 84 & 89 \\
\hline
\end{tabular}

Fuente: Investigación directa, 2013.

Es posible analizar en la tabla arriba, los resultados que se obtienen a través de los dominios y la CV, abordada de una forma general, en el instrumento de colecta de Whoqol-Bref de este estudio. De un modo abarcador, el dominio más perjudicado entre los participantes fue el físico (DF) con media de 59,44 y el más preservado fue el dominio social (DS), con media de 72,87. Los demás dominios, tanto psicológico (DP) como ambiental (DA) también presentaron medias bajas, reflejando en la CV de los portadores de IRC que presentaron una media de 64,96.

El compromiso en el DF refleja el panorama de una enfermedad crónica, donde el tratamiento genera dolor e incomodidad, así como la dependência de medicación y terapia renal sustitutiva; genera fatiga, disminuye la movilidad del paciente, ya que este precisa conectarse a una máquina durantes varios dias por semana, esto repercute en el sueño y reposo, en la actividad de la vida cotidiana y en la capacidad de trabajo.

Las condiciones ambientales también fue un dominio con media baja, demostrando que los pacientes con IRC en uso de hemodiálisis, sienten dificultades en adquirir nuevas habilidades, oportunidades de trabajo, recreación, ocio y tal vez hasta desplazarse de un lugar a otro.

De esta forma, se concluye que las limitaciones físicas ocasionadas por la enfermedad, así como las condiciones ambientales en que viven, son los factores más agravantes para la calidad de vida de los pacientes con IRC detectadas por este estudio. Llevando a creer también que, con el tiempo, los pacientes en hemodiálisis consiguen superar desafíos psicológicos impuestos, así como buscar y contar con un mayor apoyo social para el enfrentamiento de la enfermedad y su tratamiento.

Una buena característica del grupo estudiado fue la mejor media en las relaciones sociales, mostrando que, a pesar de la enfermedad y del tratamiento, los portadores de IRC mantien buena relación personal y actividad sexual y apoyo social. En lo tocante a CV, esta obtuvo una media de 64,96, un valor que no caracteriza una calidad de vida mala, por ello, al mismo tiempo, no condiciona una CV buena, y sí regular. 
Tabla 3. Correlación entre los dominios del WHOQOL-bref de pacientes en hemodiálisis (N=39). Cajazeiras-PB, 2013.

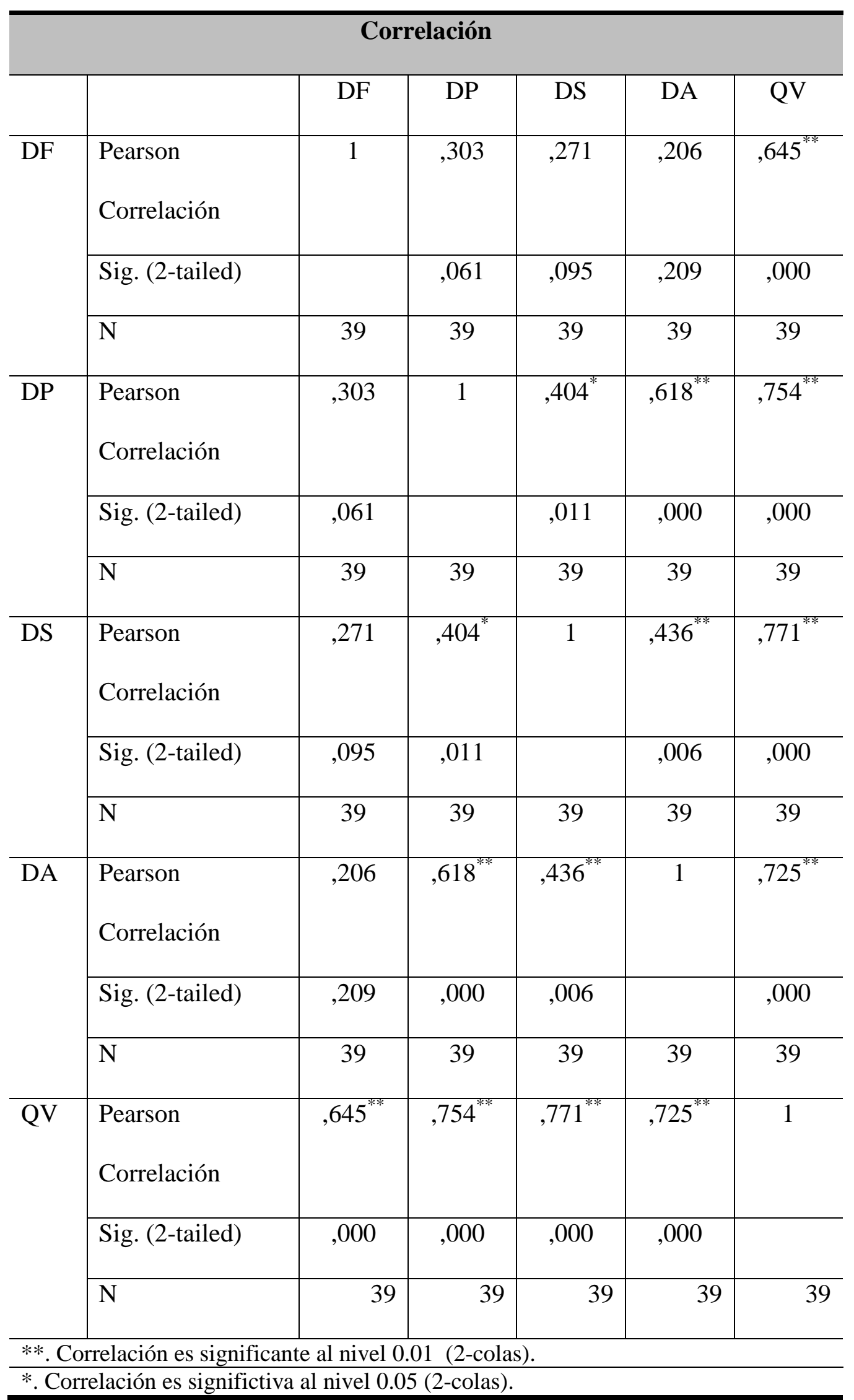

Fuente: Investigación directa. 2013. 
La tabla 3 se trata de una correlación entre los dominios y la CV. Se utilizó el test de Correlación de Pearson entre las variables, cuyo resultado indicó relación de significancia entre los diversos dominios, en que todos presentaron una correlación alta con la CV.

Refiriéndose solo a los dominios en sí, fue posible analizar que el DF, en contradicción con la literatura, en este caso, no tuvo correlación con otros dominios específicamente, solo interfiere de forma general y significativa en la CV.

La condición crónica y el tratamiento hemodialítico son fuentes de estrés, lo que ocasiona muchos problemas como aislamiento social, pérdida del empleo, dependencia de la asistencia social, parcial imposibilidad de locomoción y paseos, disminución de la actividad física, necesidad de adaptación a la pérdida de la autonomía, alteraciones de la imagen corporal y también un sentimiento ambiguo entre miedo a vivir y a morir(3).

Con relación a los demás dominios, fue posible observar que el DP y el DS poseen una pequeña correlación entre sí, por ello, cuando se trata del DA, ambos tienen fuerte influencia, lo que sugiere que las relaciones sociales, el apoyo de familiares y amigos, las cuestiones financieras y el medio en que viven esos pacientes provocan un cambio en lo psicológico individual, y esto porque las condiciones de vida son únicas y divergentes para cada persona.

\section{DISCUSIÓN}

Se sabe que con el paso de la edad, las funciones reguladoras del organismo disminuyen gradualmente, lo que evidencia la aparición de enfermedades crónicas en esta etapa de la vida. Es posible analizar que el índice de IRC afecta a más hombres que mujeres, lo que sugiere una disminución en relación con los cuidados de salud por parte de este género, perjudicando el proceso de prevención y diagnóstico precoz de las enfermedades.

El aumento de los casos de enfermedades renales se debe principalmente a las patologías agravantes y al aumento de la expectativa de vida de la población, visto que con el paso de los años, el organismo tiende a perder sus funciones reguladoras gradualmente ${ }^{(9)}$.

En un estudio realizado en 2009, hay constatación de que los entrevistados que tenían pareja estable presentaron mejor media en relación a la dimensión física, comparados con los que no tenían pareja estable, indicando que la capacidad para desempeñar las actividades diarias se volvió más fácil con la ayuda y el apoyo del compañero $^{(10)}$

Es importante destacar que, de modo general, cuanto mayor es el nivel de estudios más informaciones tendrá ese paciente sobre su condición clínica y las formas de mantener una vida más saludable.

El autocuidado puede ser considerado una práctica de funciones reguladoras que los individuos desempeñan con el objetivo de mantener un estilo de vida saludable, que no entrañe riesgos para las funciones vitales. De esta forma, colabora intensamente con la calidad de vida, una vez que el propio cuidado de la salud evita o disminuye 
conductas médicas. Por tanto, se acredita que el conocimiento es una herramienta de gran importancia y que influye en las habilidades de autocuidado de cada individuo ${ }^{(11)}$.

Adaptarse a las características de la IRC, sabiendo que no tiene cura y su tratamiento es muy complejo, significa adaptarse también a las numerosas alteraciones en la calidad de vida, debido a los varios síntomas presentados en el transcurso de la dolencia. Algunos de ellos causan diferentes niveles de limitación física y condiciones de trabajo, constituyendo un problema de salud social y económico ${ }^{(12)}$.

La convivencia social y las relaciones familiares son de gran importancia en el tratamiento hemodialítico, visto que la enfermedad acaba influyendo y generando alteraciones físicas, sociales, psíquicas y emocionales que muchas veces llevan al aislamiento del paciente y a cuadros depresivos.

Existen diferencias en las dimensiones de funcionamiento físico, efectos de la enfermedad y también función sexual, siendo más bajas las variables para las personas que vivían solas, lo que indica una peor situación de las mismas. Por tanto, destacar la importancia del soporte familiar en la CV así como su papel de colaborador con los cuidados en la salud del portador de IRC en tratamiento de hemodiálisis ${ }^{(10)}$.

El impacto ocasionado por el tiempo de hemodiálisis es poco conocido, por eso en sus estudios, se observó que los pacientes con mayor tiempo de tratamiento presentaron mejor puntuación en cuanto a los dominios de capacidad funcional, aspectos físicos, vitalidad, aspectos emocionales y aspectos sociales ${ }^{(13)}$.

La IRC acarrea para los pacientes diversas modificaciones de carácter físico y de condiciones ambientales en que viven, desencadenan alteraciones psicológicas y sociales, estando estas directamente interrelacionadas con la evaluación de la calidad de vida de los mismos. Como la percepción de la calidad de vida es única para cada participante, esta puede ser de carácter positivo, cuando el paciente busca entendimiento acerca de la enfermedad y de medidas para mejor enfrentamiento; así como de aspecto negativo, cuando existe un sentimiento de negación con relación a la enfermedad ${ }^{(14)}$.

Las restricciones ocasionadas por la hemodiálisis pueden alterar de forma significativa la CV de los portadores de IRC, una vez que las señales y síntomas físicos de la dolencia acarrean cambios en el estilo de vida y en la capacidad para el trabajo, despertando cuadros depresivos con relación a la enfermedad ${ }^{(15)}$.

Las alteraciones en los hábitos físicos pueden estar relacionadas principalmente con el factor fatiga y las señales de deterioro músculo-esquelético y debilidades físicas derivadas de la acción de la hemodiálisis, lo que desencadena otros síntomas como sentimientos negativos, depresivos o irritativos ${ }^{(15)}$.

La necesidad de realizar continuamente la hemodiálisis colabora a la dependencia de tratamiento médico, incluido en el DF, e interfiere en el trabajo y en los estudios, acarreando falta de energía y disposición para desempeñar actividades diarias.

En el DP analizado, un estudio realizado en Minas Gerais/Brasil, muestra que los resultados se asemejan. La situación de la CV de los pacientes entrevistados fue de ni mala, ni buena, lo que va en contra del resultado de la media obtenida en este 
estudio, considerando que en la evaluación, cuanto más cerca de $100 \%$, mejor el resultado. Los mismos consideraron que en las relaciones sociales los pacientes presentaron una medida entre buena y muy buena, en contra de la media obtenida en este estudio en relación al $\mathrm{DS}^{(16)}$.

En contrapartida, otro estudio muestra que el factor social es uno de los más perjudicados, al llevar al paciente muchas veces a la eliminación de las relaciones sociales $^{(17)}$.

En lo que respecta a la CV, varios aspectos como el físico, el psicológico, el social, incluyendo el nivel de satisfacción de cada uno y la importancia que se da a la vida, así como la capacidad de realizar actividades contribuyen a una CV buena o excelente ${ }^{(2)}$.

Vale destacar y observar con atención que el tiempo de tratamiento de hemodiálisis interfiere en las dimensiones del funcionamiento físico, energía y fatiga, pues los mismos alteran la capacidad de desempeñar las actividades diarias. Por ello, el tiempo de permanencia en la hemodiálisis induce al paciente a buscar formas de lidiar con las alteraciones de los dominios con el paso del tiempo, siendo este un factor favorable a una mejor calidad de vida ${ }^{(10)}$.

Por tanto, una vez que los dominios tienen correlación entre sí, la calidad de vida depende directamente de al menos un mínimo de condiciones buenas en cada uno de estos, para que el individuo pueda seguirr la vida sin mayores problemas en los hábitos de vida tras el descubrimiento de la enfermedad e inicio del tratamiento de hemodiálisis.

La enfermería tiene como principal foco el cuidado y la asistencia al paciente, y estos, van más allá de los procedimentos técnicos y requieren un establecimiento de relación cliente-profesional, a fin de entender las mayores necesidades del paciente para garantizar un trabajo humanizado, preocupado con el ser cuidado ${ }^{(18)}$.

Tratándose específicamente del sector clínico de hemodiálisis, este requiere una atencion especializada de enfermería, principalmente, para mantenimiento de la calidad de vida del cliente. De esa forma, destaca la importancia del profesional de enfermería en este sector acerca del cuidado prestado, así como la calidad de la asistencia y la educación del paciente renal crónico, con el objetivo de alcanzar una atención cualificada en el servicio, considerando el alto nivel de complejidad y de dependencia del tratamiento de hemodiálisis ${ }^{(18)}$.

\section{CONCLUSIONES}

El presente estudio posibilitó conocer la CV de los pacientes portadores de IRC en tratamiento de hemodiálisis del municipio de Cajazeiras, Estado da Paraíba; objetivando caracterizar sus variables sociodemográficas y, por medio del instrumento Whoqol-Bref, averiguar los factores que interfieren en las actividades diarias e influyen en la CV de los mismos.

La IRC es una enfermedad incurable y de progresión continua que tiene como forma de tratamiento la hemodiálisis, siendo esta una modalidad que exige disciplina y que al mismo tempo conlleva diversas alteraciones en el ámbito físico, psicológico, social y ambiental, los cuales influyen en la calidad de vida del paciente. 
Es importante destacar que, incluso con tales alteraciones, la hemodiálisis es vista como un sinónimo de mantenimiento de la vida, ya que ejerce una de las funciones vitales que es el sistema de filtración de la sangre, sin la cual no serían eliminados los residuos tóxicos e inadecuados para el organismo.

Se observó que con relación al DF, este fue el más perjudicado en términos de evaluación, concluyendo que las limitaciones impuestas por el tratamiento llevaban a los pacientes a restricciones con relación al trabajo, y a desempeñar actividades físicas rutinarias así como la necesidad de desplazamiento para garantizar el tratamiento, visto que hay una dependencia de este como garantía de vida, tornándose así un factor estresante para el paciente.

Entre el DP y DS, se observó que los mismos no fueron tan perjudicados durante la evaluación, lo que lleva a creer que los pacientes tienen un grado de aceptación elevado con relación al tratamiento, destacando también que los mismos creenn firmemente en este con esperanza, pues tienen plena consciencia de que es el único medio de continuar con vida. Aunque todavía está en duda, quedó claro que los portadores de IRC tienen relaciones sociales satisfactorias, obteniendo así el apoyo necesario para el enfrentamiento de la enfermedad así como contribuyendo a la disminución de sentimientos negativos e impotencia dentro del medio social.

El DA también presentó una media baja, concluyendo que existe un déficit de seguridad, confort, oportunidad de ocio, recursos financieros, medios de transporte, entre otros, que perjudican la calidad de vida de los pacientes en tratamiento hemodialítico. Es conveniente analizar que la satisfacción con el medio de transporte es fundamental para la locomoción al centro de hemodiálisis.

Fue posible analizar que existe, entre la mayor parte de la población, una insatisfacción con la capacidad de trabajo, una disminución en la renta que los limita a mantener el mismo patrón de vida que antes de la enfermedad; las restricciones y la disminución brusca en las actividades de ocio y paseos. Se observó también una satisfacción con las relaciones sociales y familiares, no se sentían perjudicados de forma alguna con el servicio de salud del centro de hemodiálisis, y algunos todavía con la modalidad de tratamiento, mantienen una esperanza de que la vida puede continuar, aunque para ello sean necesarios los esfuerzos y las restricciones impuestas. La busca de la CV implica buena voluntad y significa una lucha constante entre los límites impuestos por la enfermedad y su modalidad de tratamiento, así como buscar el control de los sentimientos. De ahí la importancia del apoyo social y familiar.

De un modo general, los pacientes con IRC en tratamiento hemodialítico tienen una calidad de vida regular. Es importante destacar que, incluso con todas las alteraciones acarreadas con el transcurso de la enfermedad, la población diana de este estudio demostró que, a pesar de las restricciones y de los cambios en el hábito de vida, es importante continuar y superar dificultades, objetivando una CV mejor, o sea, vivir bien, aunque la eliminación de las señales y de los síntomas sea improbable.

Como sugerencia, es necesario que los profesionales de salud busquen cada vez más informar a los portadores acerca de la enfermedad, de las condiciones individuales, de los límites impuestos por la modalidad de tratamiento y conocer las necesidades, anhelos y miedos; objetivando el esclarecimiento, favoreciendo el 
aprendizaje, para que el público diana en cuestión pueda conocer la importancia de la continuidad del tratamiento, así como desarrollar mejor autocuidado y mejor CV.

Finalizando, este estudio fue, por tanto, de gran relevancia, pues ofrece ayudas para que el equipo responsable del tratamiento de hemodiálisis perciba la necesidad e importancia de evaluar la calidad de vida de los pacientes hemodialíticos y les proporcione los medios de optimización en esa modalidad de tratamiento.

Se espera contribuir para futuros estudios, considerando que trabajar calidad de vida es complejo, sin embargo es de gran importancia en la promoción del bienestar de los portadores de IRC en tratamiento de hemodiálisis.

\section{REFERENCIAS}

1 - Martins MRI, Cesarino CB. Qualidade de Vida de Pessoas com Doença Renal Crônica em Tratamento Hemodialítico. Rev Latino-am Enfermagem, 2005 [citado 2014 Nov 12]; 13(5): [06 telas]. Disponível em: http://bvsms.saude.gov.br/bvs/is digital/is 0106/pdfs/IS26\%281\%29017.pdf

2 - Bezerra KV. Estudo do Cotidiano e Qualidade de Vida de Pessoas com Insuficiência Renal Crônica (IRC), em Hemodiálise [dissertação]. Ribeirão Preto: Universidade de São Paulo, Faculdade de Medicina de Ribeirão Preto; 2006.

3 - Machado LRC, Car MR. A Dialética da Vida Cotidiana de Doentes com Insuficiência Renal Crônica: entre o inevitável e o casual. Rev Esc Enferm USP, 2003 [citado 2014 Nov 12]; 37(3): [09 telas]. Disponível em: http://www.scielo.br/pdf/reeusp/v37n3/04.pdf

4 - Guedes KD, Guedes HM. Qualidade de Vida do Paciente Portador de Insuficiência Renal Crônica. Revista Ciência \& Saúde, Porto Alegre, 2012 [citado 2014 Nov 13]; 5(1): [06 telas]. Disponível em: http://revistaseletronicas.pucrs.br/ojs/index.php/faenfi/article/view/9734/7746

5 - Pennafort VPS, Furtado AM, Fialho AVM, et al. Produção do Conhecimento Científico de Enfermagem em Nefrologia. Rev.bras.enferm, 2010 [citado 2014 Nov 12]; 63(5): [07 telas]. Disponível em: http://www.scielo.br/pdf/reben/v63n5/22.pdf

6 - Pereira MG. Epidemiologia: teoria e prática. 12 ${ }^{\underline{a}}$ edition. Rio de Janeiro: Guanabara Koogan, 2008.

7 - Whoqol G. Whoqol-Bref. [Acesso em 12 Nov 2014]. Disponível em: http://www.ufrgs.br/psiquiatria/psiq/WHOQOL-OLD\%20Manual\%20POrtugues.pdf

8 - Sociedade Brasileira de Nefrologia. Censo de Diálise. 2011 [acesso em 13 Nov 2014]. Disponível em: http://www.sbn.org.br/

9 - Salgado Filho N, Brito DJA. Doença Renal Crônica: a grande epidemia deste milênio. J Bras Nefrol, 2006 [citado 2014 Nov 13]; 28(3): [05 telas]. Disponivel em: http://www.jbn.org.br/detalhe suplemento.asp?id=1553

10 - Cordeiro JABL, Brasil VV, Silva AMTC, et al. Qualidade de vida e tratamento hemodialítico: avaliação do portador de insuficiência renal crônica. Rev. Eletr. Enf, 2009 [citado 2014 Nov 12];11(4): [09 telas]. Disponível em: http://www.fen.ufg.br/fen revista/v11/n4/pdf/v11n4a03.pdf

11 - Baquedano IR, Santos MA, Teixeira CRS, et al. Fatores relacionados ao autocuidado de pessoas com diabetes mellitus atendidas em Serviço de Urgência no México. Rev. esc. enferm. USP, 2010 [citado 2014 Nov 10]; 44(4): [07 telas]. Disponível em: http://www.scielo.br/pdf/reeusp/v44n4/23.pdf

12 - Rudnicki T. Preditores de Qualidade de Vida em Pacientes Renais Crônicos. Estud. psicol. (Campinas), 2007 [citado 2014 Nov 12]; 24(3): [10 telas]. Disponível em: http://www.scielo.br/pdf/estpsi/v24n3/a06v24n3.pdf 
13 - Silveira CB, Pantoja IKOR, Silva ARM, et al. Qualidade de Vida de Pacientes em Hemodiálise em um Hospital Público de Belém - Pará. J. Bras. Nefrol, 2010 [citado 2014 Nov 11]; 32(1): [06 telas]. Disponível em: http://www.scielo.br/pdf/ibn/v32n1/v32n1a08.pdf

14 - Higa K et al. Qualidade de Vida de Pacientes Portadores de Insuficiencia Renal Crônica em Tratamento de Hemodilise. Acta Paul Enferm, 2008 [citado 2014 Nov 13]; 21(Número especial): [04 telas]. Disponível em: http://www.scielo.br/pdf/ape/v21nspe/a12v21ns

15 - Tretini M, Corradi EM, Araldi MAR, Tigrinho FC. Qualidade de Vida de Pessoas Dependentes de Hemodiálise Considerando Alguns Aspectos Físicos, Sociais e Emocionais. Texto \& Contexto Enfermagem, 2004 [citado 2014 Nov 15]; 13(1): [10 telas]. Disponível em: http://www.redalyc.org/pdf/714/71413111.pdf

16 - Terra FS, Costa AMDD. Avaliação da Qualidade de Vida de Pacientes Renais Crônicos Submetidos à Hemodiálise. R Enferm. UERJ, Rio de Janeiro, 2007 [citado 2014 Nov 10]; 15(3): [07 telas]. Disponível em: http://www.facenf.ueri.br/v15n3/v15n3a18.pdf 17 - Meireles VC, Goes HLF, Dias TA. Vivências Do Paciente Renal Crônico Em Tratamento Hemodialítico: Subsídios Para $O$ Profissional Enfermeiro. Ciência, Cuidado e Saúde, Maringá, 2004 [citado 2014 Nov 12]; 3(2): [10 telas]. Disponível em: http://www.periodicos.uem.br/ojs/index.php/CiencCuidSaude/article/view/5423/3475

18 - Rodrigues TA, Botti NCL. Cuidar e o Ser Cuidado na Hemodiálise. Acta Paul Enferm. 2009 [citado 2014 Nov 12]; 22(Especial-Nefrologia): [03 telas]. Disponível em: http://www.scielo.br/pdf/ape/v22nspe1/15.pdf

Recibido: 4 de diciembre 2014; Aceptado: 5 de marzo 2015

ISSN 1695-6141

(C) COPYRIGHT Servicio de Publicaciones - Universidad de Murcia 